\title{
Dinámica temporal de la riqueza de especies y la abundancia de mariposas frugívoras (Lepidoptera: Nymphalidae) en la sabana inundable del municipio de Arauca (Colombia)
}

\author{
○Juan Carlos Agudelo-Martínez*, ĐEvelin María Gómez-Tapia, @Néstor Pérez-Buitrago \\ Universidad Nacional de Colombia, Sede Orinoquia, Arauca, Colombia
}

\begin{abstract}
Resumen
El orden Lepidoptera es uno de los taxones más diversos y conocidos de insectos. Sin embargo, hay aspectos ecológicos de este grupo que se han investigado poco en la Orinoquia colombiana. En el estudio se documenta la dinámica temporal de la riqueza y la abundancia de mariposas frugívoras en la sabana inundable del municipio de Arauca y su relación con las variables climáticas de temperatura y precipitación. El muestreo se realizó a $9 \mathrm{~km}$ de la cabecera municipal de Arauca, usando seis trampas Van Someren Rydon durante cinco días al mes a lo largo de 24 meses. Se registraron 227 individuos de 21 especies. Los estimadores no paramétricos de diversidad indicaron que el muestreo tuvo una representatividad de 87,9 a 91,2 \% de las mariposas frugívoras del área. Los análisis de agrupamiento y de similitudes (ANOSIM) indicaron que la respuesta de la comunidad de mariposas corresponde al régimen unimodal de precipitación de la Orinoquia, con mayor riqueza de especies y abundancia durante los meses de lluvia. Se estableció que las temperaturas en los días de muestreo tuvieron un efecto negativo en las tasas de captura. Este trabajo representa la primera aproximación a la dinámica temporal de la riqueza y la abundancia de lepidópteros y su relación con las variables climáticas en el norte de la Orinoquia colombiana. (C) 2018. Acad. Colomb. Cienc. Ex. Fis. Nat.
\end{abstract}

Palabras clave: Estacionalidad; Mariposas diurnas; Orinoquia; Biodiversidad.

Temporal dynamics of species richness and abundance of fruit-feeding butterflies (Lepidoptera: Nymphalidae) in the flooding savannas of Arauca (Colombia)

\begin{abstract}
The insect order Lepidoptera is one of the most diverse and well known. However, the ecological aspects of this group in the Colombian Orinoquia have been poorly investigated. In this study, we documented the temporal dynamics of species richness and abundance of fruit-feeding butterflies in the flooded savanna of the municipality of Arauca. These temporal dynamics were related to the climatic variables of temperature and precipitation. The study was conducted near the town of Arauca $(9 \mathrm{~km})$. We sampled for five days per month for 24 months. We captured 227 butterflies from 21 species representing between $87.9 \%$ and $91.2 \%$ of fruit-feeding butterflies in the study area as shown by the non-parametric estimators of diversity. Cluster and ANOSIM analyses evidenced a positive relationship with rainfall resulting in greater species richness and abundances during the rainy season. There was a negative correlation between the temperatures during sampling days and the number of butterflies captured. This study is the first approach to the temporal dynamics of the richness and abundance of Lepidoptera and their relationship with climatic variables in the northern Colombian Orinoquia. (C) 2018. Acad. Colomb. Cienc. Ex. Fis. Nat.
\end{abstract}

Key words: Seasonality; Diurnal butterflies; Orinoquia; Biodiversity.

\section{Introducción}

El estudio de las interacciones entre los organismos y los factores abióticos es una de las preguntas fundamentales de la ecología (Odum, 1959; Chapin, et al., 1997). Actualmente, muchos científicos adelantan proyectos encaminados a entender dichas interacciones; en el Neotrópico algunas instituciones como la Organización de Estudios Tropicales, el Instituto Nacional de Pesca y Acuicultura (INPA) y el Instituto Smithsoniano los promueven por su importancia para la comprensión de la biodiversidad con fines de conservación, para determinar los valores que los ecosistemas proveen e, incluso, para el desarrollo de nuevas tecnologías basadas en las adaptaciones que los organismos han desarrollado frente al entorno (Godfaurd, et al., 2005).

\footnotetext{
*Correspondencia:

Juan Carlos Agudelo-Martínez; jcagudelo@gmail.com

Recibido: 15 de junio de 2018

Aceptado: 21 de agosto de 2018

Editor: Elizabeth Castañeda
} 
En Colombia los estudios ecológicos son relativamente pocos, en parte porque las políticas nacionales de investigación que rigen para las instituciones gubernamentales establecen que esta debe proveer beneficios inmediatos a la sociedad (Departamento Nacional de Planeación-DNP, 2014). Además, la megadiversidad del país ha implicado que gran parte de la comunidad científica encamine sus esfuerzos a documentar la riqueza de especies mediante listados de presencia y ausencia de especies en los ecosistemas colombianos (Arbeláez-Cortes, 2013; Mittermeir, et al., 1997). Por razones históricas, los estudios ecológicos en Colombia se han concentrado en la región Andina y la costa Caribe, donde se concentran la población general y de académicos del país, así como en la Amazonia, por su mayor visibilidad y la importancia del bosque húmedo tropical (Arbeláez-Cortés, 2013; Regalado, 2013). Sin embargo, las regiones del Chocó biogeográfico, una de las zonas más lluviosas del mundo y cuya diversidad de especies todavía está por documentarse, y la Orinoquia, con su aparente simpleza estructural (predominantemente sabanas), se han estudiado poco desde la perspectiva ecológica (ArbeláezCortés, 2013). Una de las consecuencias de esta situación es que por décadas los ecosistemas chocoanos y orinoquenses se han visto expuestos a la explotación de los recursos naturales con poco control o cautela, al margen del sentido común, de las leyes vigentes y del conocimiento científico (Peña-Pirazán, 2003). En el caso de la Orinoquia, la perspectiva es que sus paisajes seguirán expuestos a transformaciones drásticas a causa de la deforestación asociada con la explotación de hidrocarburos, la minería legal e ilegal, y la agricultura y la ganadería extensivas, marcando, así, un precedente nefasto para una región donde el estado del conocimiento de los procesos ecológicos y de los patrones de la diversidad son escasos (Agudelo \& Pérez-Buitrago, 2015; Romero-Ruiz, et al., 2011; Sánchez-Cuervo \& Aide, 2013). La Orinoquia colombiana es considerada como una de las ultimas áreas silvestres (Rivas, et al., 2002; Salinas, 2011), y, a pesar de ser el resultado de una larga historia de interacciones e interdependencia entre la naturaleza y las sociedades indígenas $\mathrm{y}$, más recientemente, de los colonos, ha sido reconocida por el Fondo Mundial para la Conservación de la Naturaleza como uno de los ocho ecosistemas estratégicos para la humanidad (Correa, et al., 2005) y un punto caliente de biodiversidad (Lasso, et al., 2011). Paradójicamente, la Orinoquia no tiene unidades de conservación (es decir, parques naturales, o reservas estatales o privadas) que representen la diversidad de ecosistemas de la región (Sánchez-Cuervo \& Aide, 2013), lo que, sumado al desconocimiento de su biodiversidad, las interacciones ecológicas y los servicios que proveen sus ecosistemas, conlleva la subestimación de su potencial estratégico y aumenta el riesgo de no poder garantizar su persistencia en el futuro (Agudelo \& Pérez-Buitrago, 2015). En este contexto, surge la necesidad de promover estudios sobre los ecosistemas de la Orinoquia para fomentar su conocimiento y fortalecer lo que podría ser una herramienta eficaz para la evaluación a mediano y largo plazo de los procesos de transformación que se llevan a cabo en los ecosistemas de la región (Correa, et al., 2005).

La Orinoquia colombiana constituye el $22 \%$ del país y su paisaje está constituido por un mosaico de ecosistemas, entre ellos bosques, bosques de galería y morichales rodeados en una matriz de sabanas de altillanura y sabanas inundables que ocupan la mayor extensión de la región (Rippstein, et al., 2001). Esa configuración espacial del paisaje obliga a muchos animales a desplazarse entre fragmentos en busca de los recursos que necesitan para suplir sus necesidades vitales (Forman, 1997), en tanto que la marcada estacionalidad climática asociada con el régimen de lluvias unimodal genera dinámicas temporales en cuanto a la disponibilidad de los recursos bióticos (refugios en el follaje, alimento) o abióticos (recurso hídrico), lo que, a su vez, podría repercutir en la abundancia y riqueza de las especies en este ecosistema de sabana inundable.

Los lepidópteros cumplen con importantes funciones ecológicas y, además, por su ciclo biológico rápido, especificidad ecológica, taxonomía relativamente estable y facilidad de muestreo e identificación se les considera buenos bioindicadores (Fagua, 1999; Brown, 1991; DeVries, et al., 1997; Bonebrake, et al., 2010). En este sentido, se plantea la hipótesis de que, dado que los sustratos para la postura de huevos y la dieta de los estadios larvales de muchas mariposas están asociados a la vegetación, y esta a su vez depende del recurso hídrico, existe una respuesta de la comunidad de las mariposas frugívoras asociada con el régimen climático. El objetivo de este estudio fue documentar la dinámica temporal de la riqueza y la abundancia de especies de mariposas frugívoras y su asociación con el régimen climático de la sabana inundable del municipio de Arauca, lo cual aportaría al conocimiento de la comunidad de lepidópteros frugívoros en la zona norte de la región de la Orinoquia colombiana.

\section{Métodos}

Area de estudio. El sitio de estudio se ubicó a $9 \mathrm{~km}$ de la cabecera municipal de Arauca (Arauca), en el campus de la Sede Orinoquia de la Universidad Nacional de Colombia. El campus tiene 50 hectáreas, de las cuales 1,5 corresponden a edificaciones rodeadas de un paisaje predominantemente compuesto por sabanas inundables $\mathrm{y}$, en menor proporción, terraplenes con bosques secundarios. La altitud es de 128 $\mathrm{m}$, presenta una temperatura media anual de $30^{\circ} \mathrm{C}$, con una humedad relativa promedio de $80 \%$ en la época lluviosa y de $65 \%$ en temporada seca. La precipitación media anual se acerca a los $2.200 \mathrm{~mm}$, con un régimen unimodal que presenta un periodo de lluvias de ocho meses comprendidos desde finales de abril hasta principios de noviembre, cuando cae el $95 \%$ de la lluvia del año, y un periodo seco de cuatro meses desde diciembre hasta marzo (Rippstein, et al., 2001).

Datos climatológicos. Los datos de los parámetros climatológicos se obtuvieron de una estación meteorológica Vantage Pro2 (Davis ${ }^{\circledR}$ Instruments Inc.), instalada en 
la sede Orinoquia desde el mes de octubre de 2013. La estación se configuró para transmitir cada hora información de temperatura, precipitación, humedad, velocidad y rumbo del viento, sensación térmica, punto de rocío e índice de calor. A partir de esta información se elaboró un climograma en el que se registraron las temperaturas promedio, máxima y mínima, así como la precipitación mensual. Los datos de clima previos a la instalación de esta estación (mayo de 2013 a septiembre de 2013) se obtuvieron de la que está instalada en el aeropuerto Santiago Pérez Quiroz, localizado a seis km del sitio de estudio.

Muestreo e identificación de especímenes. El muestreo para la recolección de mariposas frugívoras se llevó a cabo usando seis trampas Van Someren-Rydon cebadas con banano en descomposición, que se instalaron durante cinco días cada mes, desde mayo del 2013 hasta abril de 2015 (24 meses). Cada muestreo mensual representó 12 horas por trampa por día, para un total de 1.440 horas/trampa durante todo el estudio. Las trampas se distribuyeron cada 20 metros y se colgaron a una altura promedio de tres metros en los árboles de una cerca viva aledaña a uno de los estacionamientos vehiculares del campus. Las trampas se revisaban y se volvía a poner cebo entre las 16:30 y 17:30 diariamente. Todos los especímenes capturados se recolectaron y almacenaron en sobres, siguiendo la metodología propuesta por Villarreal, et al. (2006) y reposan en la Colección Entomológica de la Orinoquia (CEO) de la Sede Orinoquia de la Universidad Nacional. La identificación se llevó a cabo por comparación con diferentes fuentes bibliográficas: Warren, et al. (2013), D'Abrera $(1987,1988)$ y García, et al. (2002).

Análisis de los datos. Se emplearon los componentes alfa, beta y gamma de diversidad como una herramienta metodológica que no refleja el concepto espacial para el cual se formularon originalmente (Ricklefs, 2005), pero que permite hacer comparaciones en escalas temporales durante el muestreo, tal como fueron empleados por DeVries, et al. (1997) y DeVries \& Walla (2001). En este trabajo se consideraron de la siguiente manera: la diversidad alfa hace referencia a la diversidad puntual en cada uno de los meses de muestreo $\left(\alpha^{t}\right)$; la diversidad beta hace referencia al recambio de especies entre meses $\left(\beta^{t}\right)$, y la diversidad gama, al total de las especies observadas en los 24 meses de muestreo $\left(\gamma^{t}\right)$. La diversidad $\beta^{t}$ se calculó mediante la fórmula: 1-[log $((2 \mathrm{a}+\mathrm{b}+\mathrm{c}) /(\mathrm{a}+\mathrm{b}+\mathrm{c})) / \log 2]$ (Lennon, et al., 2001; Koleff, et al., 2003) para cada uno de los pares posibles de meses que presentaban capturas, por lo tanto, se excluyeron los meses de febrero y marzo de 2014 y el mes de marzo de 2015 de este análisis. El cálculo se llevó a cabo usando la librería Vegan (Oksanen, et al., 2016) con el programa estadístico R (R Core Team, 2003). Los resultados del cálculo de la diversidad $\beta^{t}$ se tomaron como una matriz de distancias y se representaron en un dendrograma usando como método de agrupación el enlace completo mediante la función "hclust" en el programa estadístico R (R Core Team, 2003).
Para documentar y determinar con certeza la riqueza de especies de mariposas durante los meses de muestreo, se proyectaron curvas de acumulación de especies a partir de los estimadores no paramétricos con algoritmos formulados para especies raras (Chao1: Chazdon, et al., 1998), especies únicas y especies duplicadas (Chao2: Colwell \& Coddington, 1994), especies únicas y número de muestreos (Jacknife de primer orden: Chazdon et al.,1998), especies únicas y duplicadas y el número de muestreos (Jacknife de segundo orden: Chazdon, et al., 1998), y análisis basados solo en la presencia o la ausencia (Bootstrap: Chazdon, et al., 1998). Estos estimadores se calcularon en el programa EstimateS 9.1.0 (Colwell, 2013).

Para la comparación de las temporadas, se hizo un análisis de similitud (ANOSIM) usando cada una de las temporadas climáticas como grupo. En este análisis se obtiene una prueba de significación entre los grupos con base en el cálculo de una matriz de distancias convertidas en rangos de las medidas obtenidas, en lugar de trabajar con las medidas de riqueza y abundancia obtenidas en cada uno de los meses (Clarke, 1993). En este se utilizó la función "anosim" de la librería Vegan (Oksanen, et al., 2016) ejecutada mediante el programa estadístico R (R Core Team, 2003).

Para relacionar la abundancia y la riqueza de especies con los factores climáticos se usó la correlación de Spearman, específicamente con los parámetros de temperatura mínima, temperatura máxima y temperatura media, considerando únicamente las horas de sol $(6: 00 \mathrm{am}-6: 00 \mathrm{pm})$ durante los días de muestreo de cada mes. La relación de la riqueza y la abundancia de mariposas con la precipitación se evaluó con el total de la precipitación mensual con el programa PAST 2.17C (Hammer, et al., 2001).

Para comparar cada uno de los ciclos de doce meses se realizó una prueba de t pareada (Sokal \& Rohlf, 1979), con el fin de evaluar si existían diferencias estadísticamente significativas con respecto a la riqueza y la abundancia de especies tomando dos ciclos de doce meses cada uno (ciclo 1: mayo de 2013 a abril de 2014, ciclo 2: mayo de 2014 a abril de 2015).

\section{Resultados y discusión}

Durante los 24 meses de muestreo se registraron 227 individuos pertenecientes a 21 especies y 20 géneros de la familia Nymphalidae. Las subfamilias representadas fueron Biblidinae (28,6 \%), Satyrinae (23,8 \%), Nymphalinae $(23,8 \%)$ Charaxinae (14,3 \%), y Morphinae (9,5\%). Las especies más representativas en el muestro fueron: Hamadryas feronia (Linnaeus, 1758) (26,9 \%), Callicore pitheas (Latreille, 1813) (20,7\%), Temenis laothoe (Cramer, 1777) (9,7 \%), Hermeuptychia sp1 (8,4\%), Taygetis laches (Fabricius, 1793) y Zaretis ellops (Ménétries, 1855) (5\% cada una) y Hermeuptychia hermes (Fabricius, 1775) (4,8 \%) (Figura 1) (Tabla 1). Además, se registraron 14 especies con abundancias menores de diez individuos, que representaron el $19 \%$ de los especímenes capturados durante el estudio. 
Diversidad alfat. El mes con mayor número de especies fue octubre de 2013, con nueve especies, seguido de septiembre y noviembre del mismo año, y de octubre y de diciembre de 2014, con ocho especies cada uno. Estos

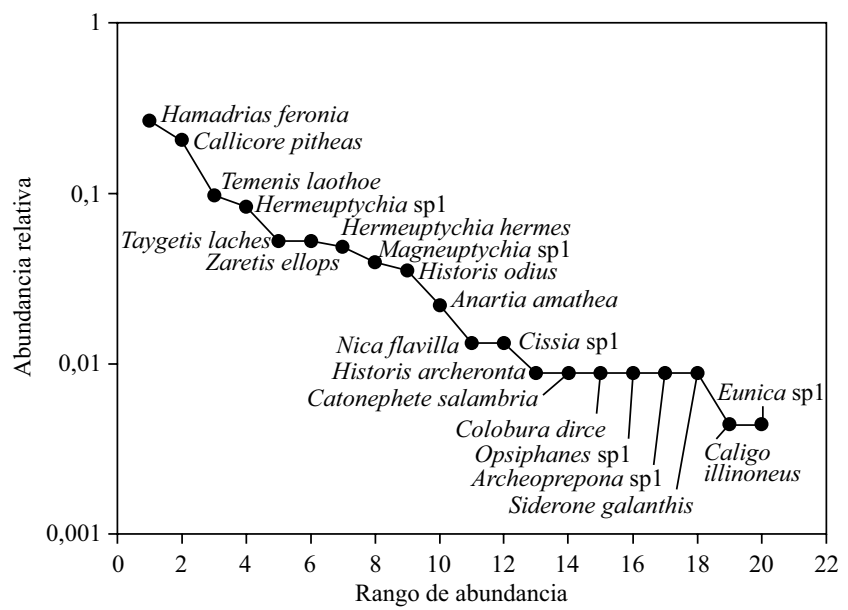

Figura 1. Curva de rango de la abundancia de especies de mariposas frugívoras recolectadas durante 24 meses en Arauca, Colombia

Tabla 1. Listado de especies recolectadas y su presencia en cada una de las temporadas climáticas

\begin{tabular}{|c|c|c|}
\hline Especie & Invierno & Verano \\
\hline Callicore pitheas (Latreille, 1813) & 45 & 2 \\
\hline Hamadrias feronia (Linnaeus, 1758) & 51 & 10 \\
\hline Hermeuptychia hermes (Fabricius, 1775) & 10 & 1 \\
\hline Historis archeronta (Fabricius, 1775) & 1 & 1 \\
\hline Nica flavilla (Godart, [1824]) & 3 & 0 \\
\hline Temenis laothoe (Cramer, 1777) & 21 & 1 \\
\hline Historis odius (Fabricius, 1775) & 8 & 0 \\
\hline Caligo illioneus (Cramer, 1775) & 1 & 0 \\
\hline Taygetis laches (Fabricius, 1793) & 12 & 0 \\
\hline Zaretis ellos (Ménétriés, 1855) & 10 & 2 \\
\hline Anartia amathea (Linnaeus, 1758) & 4 & 1 \\
\hline Catonephele salambria (Hewitson, 1852) & 1 & 1 \\
\hline Colobura dirce (Linnaeus, 1758) & 2 & 0 \\
\hline Magneuptychia sp1 & 7 & 2 \\
\hline $\begin{array}{l}\text { Opsiphanes cassina (C. Felder y } \\
\text { R. Felder, 1862) }\end{array}$ & 2 & 0 \\
\hline Siproeta stelenes (Linnaeus, 1758) & 1 & 0 \\
\hline Hermeuptychia sp1 & 17 & 2 \\
\hline Cissia sp1 & 1 & 2 \\
\hline $\begin{array}{l}\text { Archeoprepona amphimachus } \\
\text { (Fabricius, 1775) }\end{array}$ & 2 & 0 \\
\hline Eunica sp1 Hübner, [1819] & 1 & 0 \\
\hline Siderone galanthis (Cramer, 1775) & 0 & 2 \\
\hline
\end{tabular}

meses, con excepción de diciembre de 2014, corresponden a la temporada de lluvias. Por otra parte, los meses de febrero y marzo de 2013, y de marzo de 2015 fueron los menos diversos y no se registraron capturas, lo cual refleja las condiciones climáticas extremas durante la temporada de sequía.

Diversidad betat . El análisis de similitud llevado a cabo

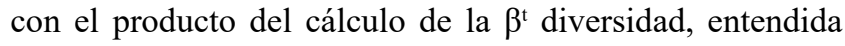
como el recambio de especies entre cada uno de los meses, generó la formación de cuatro grupos (Figura 2) con un $90 \%$ de diferencia que, de izquierda a derecha en esta Figura corresponden, primero, a los meses de enero de 2014 y enero de 2015 (temporada de sequía); segundo, al mes julio de 2013 (temporada de lluvias); tercero, a los meses de abril de 2014 y 2015 , diciembre y mayo de 2013 y febrero de 2015 (meses de sequía, excepto mayo) y, por último, los meses restantes que corresponden a la temporada de lluvias, con excepción de diciembre de 2014. Los grupos formados en este análisis reflejaron la tendencia de la riqueza y la abundancia de especies a lo largo del año; así, los meses con mayores abundancias, riqueza y recambio de especies correspondieron al grupo de los meses de lluvias, en tanto que los meses de menor valor en estos parámetros fueron de sequía. Según el análisis de similitud (ANOSIM) hubo una diferencia estadísticamente significativa entre las épocas de lluvias y sequía $(\mathrm{p}=0,032)$, lo cual confirma la tendencia observada en el análisis anterior.

Diversidad Gammat. Las curvas de acumulación de especies evidenciaron entre 21 y 24 especies usando diferentes estimadores no paramétricos. Para los 24 eventos de muestreo, las 21 especies recolectadas representaban el $97,6 \%$ de las especies calculadas por el estimador Jacknife de segundo orden. Los estimadores no paramétricos de riqueza Chao1 y Chao2 predijeron valores de riqueza de

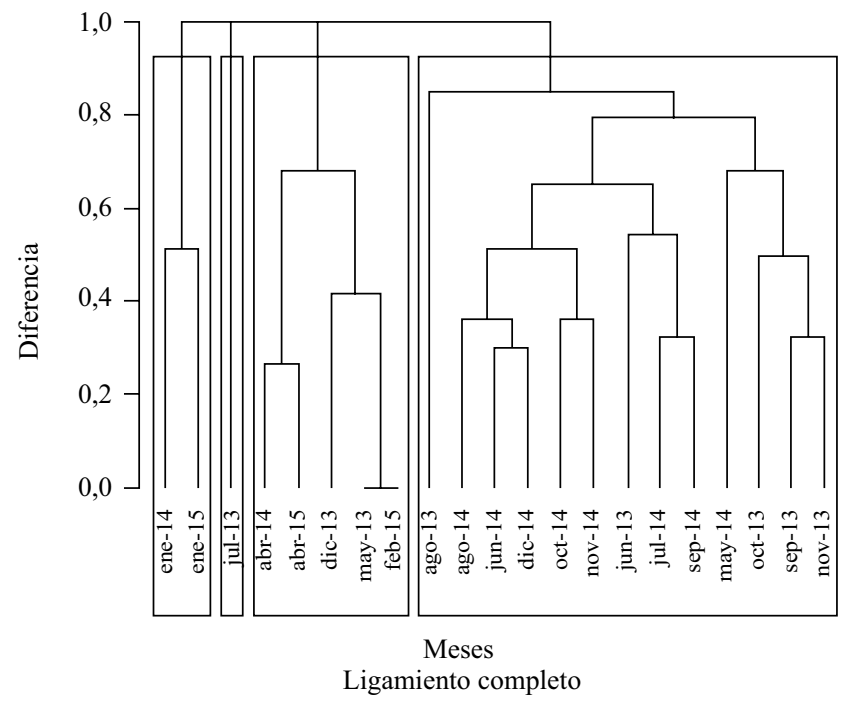

Figura 2. Dendograma para los meses de muestreo con capturas de mariposas frugívoras en Arauca. Los recuadros indican diferencias del $90 \%$ entre los grupos formados. 
21,4 y 21,3 , respectivamente, lo cual significa que las muestras recolectadas representaban entre el $98 \%$ y el $98,3 \%$ de las especies predichas por ellos. Además, con el estimador Bootstrap se calcularon 23 especies, lo que indica que se obtuvo el $91,2 \%$ de las especies predichas por el estimador. Por último, en el estimador Jacknife de segundo orden se presentó un valor estimado de 23,9 , u 87,9\% de las especies esperadas en el área de estudio (Figura 3). Así, independientemente del algoritmo usado para el cálculo del valor esperado para la riqueza de especies, los muestreos reflejaron una buena representatividad de las mariposas frugívoras que habitan la sabana inundable del municipio de Arauca.

Este resultado es comparable con el estudio de Urbano, et al. (2014), quienes reportaron 32 de especies de Nymphalidae en dos localidades del departamento de Casanare, cuyas características paisajísticas son comparables con los ambientes de sabana inundable y con parámetros abióticos similares, como la temperatura y el régimen unimodal de lluvias, de la Orinoquia. La diferencia de $34,4 \%$ en este resultado es atribuible a la diferencia en el tipo de técnicas usadas en cada uno de los trabajos, pues Urbano, et al. (2014) usaron dos técnicas complementarias (trampas Van Someren Rydon y red entomológica), en tanto que en este estudio solo se emplearon trampas Van Someren Rydon.

En la región de la Orinoquia se han reportado 158 especies de mariposas diurnas, de las cuales 100 pertenecen a la familia Nymphalidae (Andrade, 2002). En este estudio se registró el $21 \%$ de esa riqueza de especies de la comunidad de Nymphalidae, las cuales pueden considerarse importantes en el ecosistema de sabana inundable característico del área de estudio, siendo un taxón que ocupa una gran diversidad de hábitats con amplios rangos de distribución en el trópico (DeVries, et al., 1997; Chacón \& Montero, 2007). A nivel de subfamilias, los valores de riqueza encontrados para Biblidinae (seis especies) y Nymphalinae (cinco especies) reflejan su alto grado de versatilidad (Orozco, et al., 2009), el cual les permite explotar los recursos de forma generalista, en diferentes estratos vegetales y con variedad de estrategias contra los depredadores (Vargas, et al., 2011).

La riqueza de las especies y la abundancia registraron una estrecha relación $(\mathrm{r}=0,7985 ; \mathrm{p}<0,0001 ; \mathrm{n}=24)$. La riqueza de especies promedio por mes fue de 4,12 ( $\pm 2,9$; rango: $0-9$ especies), en tanto que la abundancia fue, en promedio, de 9,4 individuos ( $\pm 9,5$; rango: $0-30$ individuos) y se concentró en los meses de lluvias (abril - octubre) (Minorta-Cely \& Rangel, 2014), cuando se recolectaron 178 especímenes, es decir, el $78 \%$ del total de mariposas capturadas en el estudio (Figura 4). La prueba de t pareada usada para evaluar si existió variación en la riqueza o la abundancia en los dos ciclos de 12 meses documentados, indicó que, globalmente, los dos años fueron similares, pues no se detectaron diferencias significativas (abundancia: $\mathrm{U}=0,046 ; \mathrm{p}=0,9641 ; \mathrm{gl}=11$. riqueza: $\mathrm{U}=1,0235$; $\mathrm{p}=0,328 ; \mathrm{gl}=11$ ).

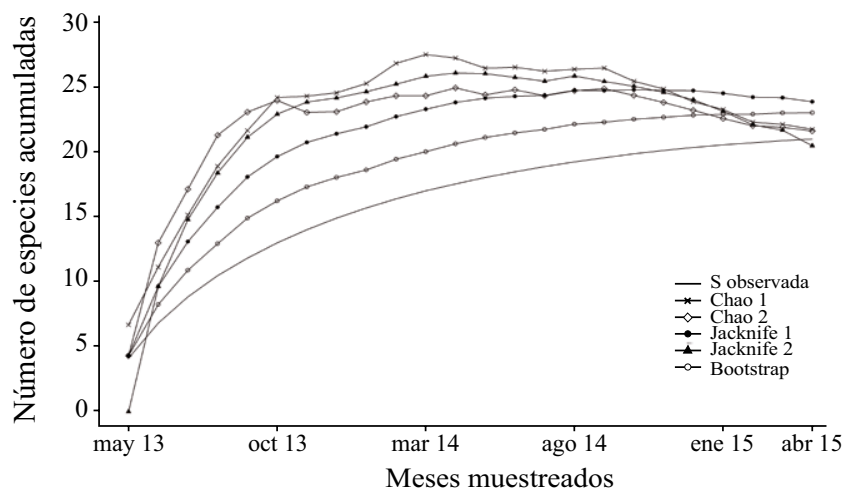

Figura 3. Curvas de acumulación de especies y estimadores no paramétricos de riqueza para los muestreos de mariposas frugívoras realizados entre mayo de 2013 y abril de 2015 en el área muestreada

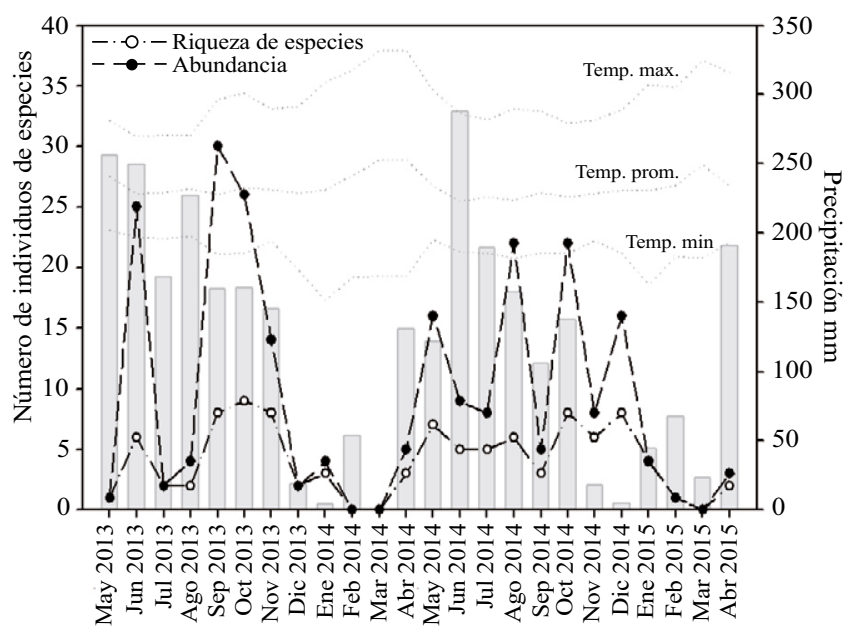

Figura 4. Abundancia y riqueza de especies de mariposas frugívoras durante los 24 meses de muestreo.

Los análisis de correlación de Spearman evidenciaron una tendencia positiva de la precipitación con respecto a la riqueza $(\mathrm{r}=0,39 ; \mathrm{p}=0,0875 ; \mathrm{n}=24 ; \mathrm{gl}=22)$ y una relación significativa de la precipitación con la abundancia $(\mathrm{r}=0,5$; $\mathrm{p}=0,00262 ; \mathrm{n}=24 ; \mathrm{gl}=22$ ). Este patrón de respuesta de la comunidad de mariposas a la precipitación concuerda con lo reportado en múltiples estudios (DeVries, et al., 1997; Shahabuddin \& Terborgh, 1999; Hilt, et al., 2007; Luna, et al., 2010; Hussain, et al., 2011; Vargas, et al., 2011; Grotan, et al., 2012; Sorto, 2013; Brito. et al., 2014; Kishimoto-Yamada \& Itioka, 2015; Franzen, et al., 2017; Habel, et al., 2018). El mecanismo que subyace a este patrón es que la disponibilidad del recurso hídrico tiene un efecto en la fenología, induciendo en la vegetación la producción de nuevos brotes de hojas y, posteriormente, de flores y frutos (Pollard, 1988). En el caso de las mariposas, esto implica una mayor oferta de alimento durante la fase larval $\mathrm{y}$, debido al aumento de la cobertura vegetal, una mayor disponibilidad de refugios (Grotan, et al., 2012; Valtonen, et al., 2013). 
Asimismo, los análisis de correlación indicaron que hubo un efecto negativo en la abundancia y la riqueza de especies asociado con las temperaturas máximas (riqueza: $\mathrm{r}=-0,62 ; \mathrm{p}=0,0033$; abundancia: $\mathrm{r}=-0,64 ; \mathrm{p}=0,02$ ) y temperaturas promedio (riqueza: $\mathrm{r}=-0,52 ; \mathrm{p}=0,02$; abundancia: $\mathrm{r}=-$ 0,$6 ; \mathrm{p}=0,005$ ) durante los días y horas de muestreo. A pesar de que muchas especies de mariposas diurnas poseen mecanismos de termorregulación que inciden en sus periodos y actividades diarias de forrajeo, búsqueda de pareja, postura de huevos y reducción del riesgo de depredación (Kingsolver, 1985; Shelly \& Ludwig, 1985; Chai \& Srygley, 1990; Clench, 1966; Sparks \& Yates, 1997; Bandini, et al., 2010), las máximas temperaturas en el sitio de estudio pudieron ser excesivas en los días de muestreo (media $=33,0 \pm 1,8$; rango: 30,8 a $37,5^{\circ} \mathrm{C}$ ). En este sentido, son relativamente pocos los estudios hechos sobre la ecología de la termorregulación en mariposas tropicales, pero Fischer, et al., (2010) y Karl, et al. (2011) usaron en experimentos de laboratorio una temperatura de $34{ }^{\circ} \mathrm{C}$ en los tratamientos con temperaturas elevadas para mariposas tropicales. Las temperaturas altas pueden tener efectos negativos en la riqueza y la abundancia de mariposas, ya que pueden causar estrés fisiológico y ello restringe los periodos de actividad (Roy, et al., 2001), afecta negativamente el sistema inmunitario (Karl, et al., 2011), y reduce la supervivencia y la fecundidad (Kingsolver \& Watt, 1983). Además, Vargas, et al., (2011) plantearon que en los meses de poca pluviosidad la pérdida de follaje en la vegetación ocasiona mayor incidencia de la radiación solar sobre la superficie del suelo, incrementando las temperaturas del sotobosque y disminuyendo la actividad de muchos lepidópteros diurnos.

Los patrones de abundancia de las especies detectados a lo largo del estudio son variados. Los picos de abundancia de algunas de ellas parecen reflejar la existencia de los ciclos de vida multivoltinos en especies como $H$. feronia y $C$. pitheas, con registros de abundancias relativamente consistentes a lo largo del año, especialmente, durante los meses en que ocurren las mayores precipitaciones en la sabana inundable de la Orinoquia (Figura 5). Aunque en menor grado, el multivoltinismo también fue evidente en $T$. laothoe, a pesar de la ausencia de capturas en los meses de sequía, lo que concuerda con lo reportado por Hernández-Mejía, et al., (2008) en México. El multivoltismo, es decir la aparición de varias generaciones a lo largo del año, se ha relacionado con la sincronización generacional frente a las condiciones ambientales y la disponibilidad de recursos durante los diferentes estadios del clico de vida de las mariposas (Wolda, 1988; Hernández-Mejía, et al., 2008). Shapiro (1974) sugirió que el multivoltinismo ocurre en especies con estrategias polífagas generalistas, lo que les permite sobrevivir y estar en la comunidad a lo largo del año, incluso en densidades poblacionales relativamente bajas, como es el caso durante los meses de sequía para especies como $H$. feronia, y C. pitheas.

Los datos sobre la abundancia también indican que algunas especies como Hermeuptychia sp1 (Figura 6) estuvo ausente en el primer año de muestreo y solo se registró en el segundo, alcanzando la cuarta mayor abundancia $(8 \%)$ de todas las especies registradas en el estudio. Ese comportamiento, aparentemente univoltino, podría deberse a lo planteado por Shapiro (1974) en cuanto a que el univoltinismo está asociado a especies monófagas. Otro factor que puede afectar negativamente las poblaciones de mariposas es que las lluvias tienen efectos sobre la fenología vegetal, y modifican la naturaleza y la concentración

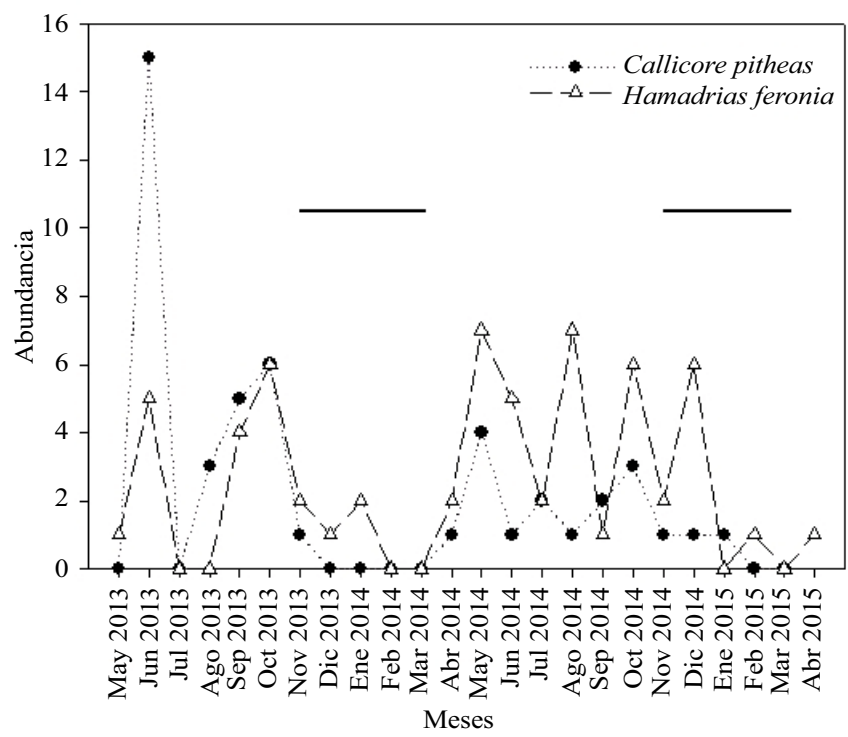

Figura 5. Variación en la abundancia de $C$. phiteas y H. feronia durante los 24 meses de muestreo. Las líneas horizontales representan los meses de sequía.

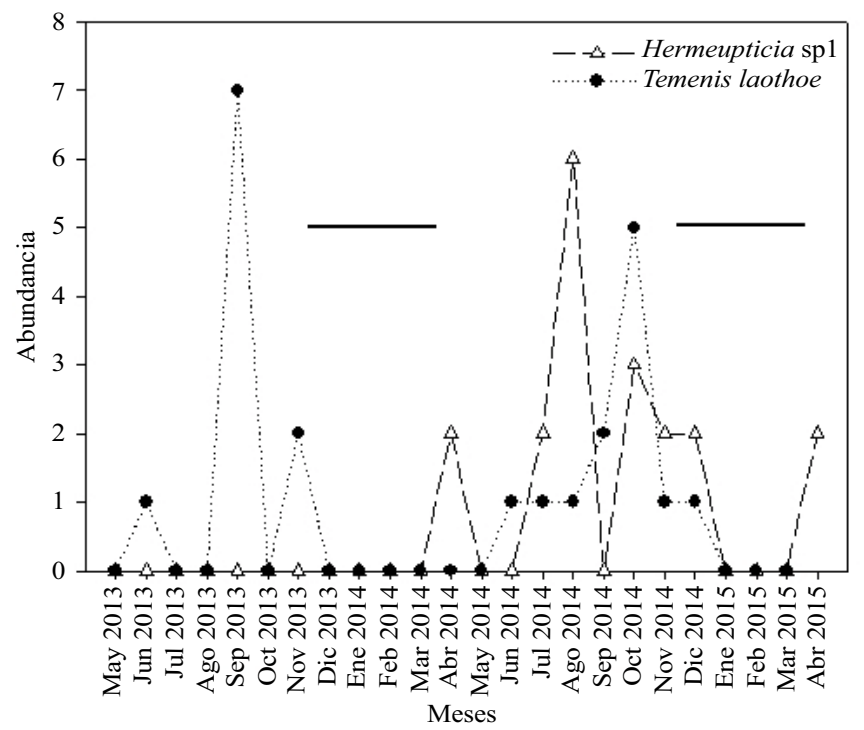

Figura 6. Abundancias de T. laothoe y Hermeuptychia sp1 en los 24 meses de estudio. Las líneas horizontales representan los meses de sequía. 
de algunos compuestos presentes en las plantas (Scott $\boldsymbol{\&}$ Epstein, 1987). Por esta razón, a lo largo del año hay meses que no son aprovechables nutricionalmente por los estadios inmaduros de algunas especies y el desarrollo de sus ciclos de vida responden a estas fluctuaciones (Hernández-Mejía, et al., 2008). Por último, los patrones de abundancia de cada población también podrían reflejar aspectos específicos relacionados con la historia natural de cada especie, los cuales no fueron considerados en la presente investigación.

\section{Conclusiones}

Este trabajo es la primera aproximación a la ecología de las mariposas frugívoras en el ambiente de sabana inundable del norte de la Orinoquia colombiana, y es novedoso también por la escala bianual que documenta. Se determinó que hay relación entre factores abióticos como la temperatura y la precipitación y la abundancia y riqueza de las especies de mariposas frugívoras en una escala temporal relativamente amplia. La respuesta de las mariposas frugívoras a los factores abióticos refleja la capacidad de algunas especies para sobrellevar condiciones ambientales mediante estrategias como la aparición de varias generaciones a lo largo del año (multivoltinismo), lo cual les permite explotar de forma más efectiva los recursos alimenticios disponibles.

Una de las implicaciones de la estrecha relación de la riqueza de especies con la estacionalidad tiene que ver con el diseño de futuros inventarios de la diversidad de las mariposas en la Orinoquia, dado que hacerlo exclusivamente durante la época seca puede subestimar la diversidad local y regional en términos de la riqueza de especies y su abundancia. El presente estudio funciona como un referente para la planeación e implementación de otros similares en esta región del país.

\section{Agradecimientos}

Los autores de este trabajo agradecen a los alumnos Ángel Matiz, Yilmer Mina, Nelly Carolina Torres Rivera, Sebastián Peralta Pérez, Maikol Jiménez, Carlos Gamba, Miguel Ángel Martínez Quiroga, Neisdan Iván Serrano Pardo, Liseth Andrea Prieto Torres, Yonatan Moreno Casteblanco y Nixon Andrés Castillejo Lozano, por su trabajo en la curaduría y la captura de buena parte de los especímenes relacionados en este artículo.

\section{Contribución de los autores}

Juan Carlos Agudelo Martínez: formulación de la idea general, recolección de muestras, identificación taxonómica, análisis de datos y preparación del manuscrito. Evelin María Gómez Tapia: recolección de muestras, identificación taxonómica, análisis de datos y preparación del manuscrito. Néstor Pérez-Buitrago: formulación de la idea general, análisis de datos, preparación y revisión del manuscrito

\section{Conflicto de intereses}

Los autores declaran que no tienen conflicto de intereses.

\section{Referencias}

Agudelo, J.C., Pérez-Buitrago, N. (2015). Notas acerca de la distribución de Papilionidae (Lepidóptera: Papilionoidea) en el norte de la Orinoquia colombiana. Bol. Cient. Mus. Hist. Nat. U. de Caldas. 19: 203-214.

Andrade, G.M. (2002). Biodiversidad de las mariposas (Lepidóptera: Rhopalocera) de Colombia. Proyecto de Red Iberoamericana de Biogeografía y Entomología Sistemática. Lepidóptera Tercer Milenio. SEA, España. 2: 153-172.

Arbeláez-Cortés, E. (2013). Knowledge of Colombian biodiversity: Published and indexed. Biodivers Conserv. 22: 2875-2906.

Bandini, R.D., Prado P.I., Brown, K.S., Freitas A.V. (2010). Temporal Diversity Patterns and Phenology in Fruit-feeding Butterflies in the Atlantic Forest. Biotropica. 42: 710-716.

Bonebrake, T.C., Ponisio, L.C., Boggs, C.L, Ehrlich P.R. (2010). More than just indicators: A review of tropical butterfly ecology and conservation. Biological Conservation. 143: 1831-1841.

Brown, K. (1991). Conservation of Neotropical Environments: Insects as Indicators. En: Collins N., Thomas J., editores. The Conservation of Insects and Their Habitats. London, Reino Unido: Academic Press Limited. p. 349-404.

Brito, M.M., Ribeiro, D.B., Raniero, M., Hasui, E., Ramos, F.N., Arab, A. (2014). Functional composition and phenology of fruit-feeding butterflies in a fragmented landscape: Variation of seasonality between habitat specialists. J Insect Conserv. 18: 547-560.

Chai, P., Srygley, R.B. (1990). Predation and the flight, morphology, and temperature of Neotropical rain forest butterflies. Rev. The American Naturalist. 135: 748-765.

Chacón, I., Montero, J. (2007). Mariposas de Costa Rica. Costa Rica: Editorial INBio. p. 366.

Chapin, F. S., Walker, B. H., Hobbs, R. J., Hooper, D. U., Lawton, J. H., Sala, O. E., Tilman, D. (1997). Biotic control over the functioning of ecosystems. Science. 277: 500-504.

Chazdon, R.L., Colwell, R.K., Denslow, J.S., Guariguata, M.R. (1998). Statistical methods for estimating species richness of woody regeneration in primary and secondary rain forests of NE Costa Rica. In: Dallmeier, F., Comiskey, J.A. Editores. Forest biodiversity research, monitoring and modelling: Conceptual background and Old World case studies. Paris, France: Parthenon Publishing. p. 285-309.

Clarke, K.R. (1993). Non-parametric multivariate analysis of changes in community structure. Australian Journal of Ecology. 18: 117-143.

Clench, H.K. (1966). Behavioral thermoregulation in butterflies. Ecology. 47: 1021-1034.

Colwell, R.K., Coddington, J.A. (1994). Estimating terrestrial biodiversity through extrapolation. Philosophical Transactions of the Royal Society of London. 345: 101- 118.

Colwell, R. (2013). Estimates: Statistical estimation of species richness and shared species from samples. Versión 9. Fecha de consulta: enero-junio de 2015. Disponible en: http://purl. oclc.org/estimates, accedida entre enero y junio de 2015.

Correa, H.D., Ruiz, S.L., Arévalo, L.M. (2005). Plan de acción en biodiversidad de la cuenca del Orinoco - Colombia / 2005 - 2015 - Propuesta Técnica. Bogotá D.C. Corporinoquia, Cormacarena, IAvH, Unitrópico, Fundación Omacha, Fundación Horizonte Verde, Universidad Javeriana, Unillanos, WWF Colombia, GTZ. Bogotá, Colombia: ARFO editores. p. 273. 
D'abrera, B. (1987). Butterflies of the Neotropical Region. Part IV. Nymphalidae (partim). Victoria, Australia: Hill House. p. 526-678.

D'abrera, B. (1988). Butterflies of the Neotropical Region. Part V. Nymphalidae (conc.) \& Satyridae. Victoria, Australia: Hill House. p. 679-877.

DeVries, P.J., Murray, D., Lande, R. (1997). Species diversity in vertical, horizontal, and temporal dimensions of a fruitfeeding butterfly community in an Ecuadorian rainforest. Biol. J. Linn. Soc. 62: 343-364.

DeVries, P.J., Walla, T.R. (2001). Species diversity and community structure in Neotropical fruit-feeding butterflies. Biol. J. Linn. Soc. 74: 1-15.

Departamento Nacional de Planeación. (2014). Bases del plan Nacional de Desarrollo 2014-2018. Bogotá D.C. Colombia. DNP.

Fagua, G. (1999). Variación de las mariposas y hormigas de un gradiente altitudinal de la cordillera Oriental (Colombia). Revista de la Academía Colombiana de Ciencias Exactas, Físicas y Naturales. 2: 75-90.

Fischer, K., Dierks, A., Franke, K., Geister, T.L., Liszka, M., Winter, S., Pflicke, C. (2010). Environmental effects on temperature stress resistance in the tropical butterfly Bicyclus anynana. PLoS ONE. 5: e15284.

Forman, R. (1997). Land mosaics: The ecology of landscapes and regions. Cambridge, United Kingdom, Cambridge University Press. p. 632.

Franzen, M., Schrader, J., Sjöberg, G. (2017). Butterfly diversity and seasonality of Ta Phin mountain area (N. Vietnam, Lao Cai province). J Insect Conserv. 21: 465-475.

García, C., Constantino, L., Heredia, M., Kattan, G. (2002). Mariposas comunes de la cordillera Central de Colombia. Wildlife Conservation Society. Feriva editores, Cali. Colombia. p. 120.

Godfaurd, J., Clements-Croome, D., Jeronimidis, G. (2005). Sustainable building solutions: A review of lessons from the natural world. Building and Environment. 40: 319-328

Gørtan, V., Lande, R., Engen, S., Sæther, B.E., DeVries, P.J. (2012). Seasonal cycles of species diversity and similarity in a tropical butterfly community. Journal of Animal Ecology. 81: 714-723.

Habel, J.C., Seibold, S., Ulrich, W., Schmitt, T. (2018). Seasonality overrides differences in butterfly species composition between natural and anthropogenic forest habitats. Anim Conserv. doi:10.1111/acv.12408

Hammer, O., Harper, D., Ryan, P. (2001). PAST: Paleontological Statistics Software Package for Education and Data Analysis. Paleontología Electrónica. 4: 9.

Hernández-Mejía, C., Llorente., J, Vargas, I., Luis A. (2008). Las mariposas (Hesperioidea y Papilionoidea) de Malinalco, Estado de México. Revista Mexicana de Biodiversidad. 79: 117-130.

Hilt, N., Brehm, G., Fiedler, K. (2007). Temporal dynamics of rich moth ensembles in the Montane Forest Zone in Southern Ecuador. Biotropica. 39: 94-104.

Hussain, K.J., Ramesh, T., Satpathy, K.K., Selvanayagam, M. (2011). Seasonal dynamics of butterfly population in DAE Campus, Kalpakkam, Tamil Nadu, India. Journal of Threatened Taxa. 3: 1401-1414.

Karl, I., Stoks, R., Block, M., Janowitz, S.A., Fischer, K. (2011). Temperature extremes and butterfly fitness: conflicting evidence from life history and immune function. Global Change Biology. 17: 676-687.
Kingsolver, J.G. (1985). Butterfly thermoregulation: Organismic mechanisms and population consequences. Journal of Research on the Lepidoptera. 24: 1-20.

Kingsolver, J.G., Watt, W.B. (1983). Thermoregulatory strategies in Colias Butterflies: Thermal stress and the limits to adaptation in temporally varying environments. The American Naturalist. 121: 32-55.

Kishimoto-Yamada, K., Itioka, T. (2015). How much have we learned about seasonality in tropical insect abundance since Wolda (1988)? Entomological Science. 18: 407-419.

Koleff, P., Gaston, K.J., Lennon, J.J. (2003). Measuring beta diversity for presence-absence data. Journal of Animal Ecology. 72: 367-382.

Lamas, G. (2004). Atlas of Neotropical Lepidóptera. Checklist: Part 4A. Hesperioidea - Papilionoidea. ATL + Scient. Publ. Gainesville, Florida, USA: Scientific Publishers. p. 439.

Lasso, C., Rial, A., Matallana, C., Ramírez, W., Señaris, J., Díaz, A., Corzo, G., Machado, A. (2011). Biodiversidad de la Cuenca del Orinoco. II Áreas prioritarias para la conservación y el uso sostenible. Instituto de investigación de recursos biológicos Alexander von Humboldt, Ministerio de Ambiente, Vivienda y Desarrollo Territorial, WWF Colombia, Fundación Omacha, Fundación La Salle de Ciencias Naturales e Instituto de Estudios de la Orinoquia (Universidad Nacional de Colombia). Bogotá, D.C., Colombia. p. 304.

Lennon, J.J., Koleff, P., Greenwood, J.J., Gaston, K.J. (2001). The geographical structure of British bird distributions: diversity, spatial turnover and scale. Journal of Animal Ecology. 70: 966-979.

Luna, M., Llorente, J., Martínez, A., Vargas, I. (2010). Composición faunística y fenología de las mariposas (Rhopalocera: Papilionoidea) de Cañón de Lobos, Yautepec, Morelos, México. Revista Mexicana de Biodiversidad. 81: 315-342.

Minorta-Cely, V., Rangel, C.J. (2014). El clima de la Orinoquia Colombiana. Colombia Diversidad Biótica XIV. La región de la Orinoquia de Colombia, Instituto de Ciencias Naturales, Universidad Nacional de Colombia. Bogotá, D.C., Colombia. p. 533-612.

Mittermeir, R., Robles, P., Mittermeir, C. (1997). Megadiversidad. Los países biológicamente más ricos del mundo. Cemex. México. p. 501.

Odum, H. T. (1959). Fundamentals of ecology. Philadelphia. United States of America. W. B. Saunders Company. p. 383.

Oksanen, J., Blanchet, F.G., Kindt, R., Legendre, P., Minchin, P.R., O'hara, R.B., Simpson, G.L., Solymos, P., Stevens, M.H.H., Wagner, H. (2016). Community Ecology Package. Versión 2.3-3. Fecha de consulta: enero-junio de 2015. Disponible en: https://github.com/vegandevs/vegan

Orozco, S., Muriel, S.B., Palacio, J. (2009). Diversidad de lepidópteros diurnos en un área de bosque seco tropical del occidente antioqueño. Actualidades Biológicas. 31: 31-41.

Peña-Pirazán, J.D. (2003). Minería y medio ambiente en Colombia. Una síntesis histórica de la mala administración de nuestros recursos naturales mineros de la degradación ambiental y de la generación conflictos sociales, asociados a desastres naturales. Una mirada hacia el futuro económico y ambiental de este importante sector productivo. Tesis de Especialización, Universidad Sergio Arboleda, Bogotá.

Pollard, E. (1988). Temperature, rainfall and butterfly numbers. Journal of Applied Ecology. 25: 819-828. 
R Core Team. (2013). R: A language and environment for statistical computing. R Foundation for Statistical Computing, Viena, Austria. Fecha de consulta: enero - junio de 3015. Disponible en: http://www.R-project.org/

Regalado, A. (2013). Venturing back into Colombia. Science. 341: 450-452.

Ricklefs, R.E. (2005). Historical and ecological dimensions of global patterns in plant diversity. Biol. Skr. 55: 583-603.

Rippstein, G., Escobar G., Motta, F. (2001). Agroecología y biodiversidad de las sabanas en los Llanos Orientales de Colombia. Cali, Colombia: Centro Internacional de Agricultura Tropical: Publicación CIAT. p. 302.

Rivas, J., Rodríguez, J., Mittermeier, C. (2002). Los llanos. En: Robles, G.P. Áreas silvestres: Las últimas regiones vírgenes del mundo. México: Cemex, Conservación Internacional. p. 576.

Romero-Ruiz, M.H., Flantua, S.G.A., Tansey, K., Berrio, J.C. (2011). Landscape transformations in savannas of northern South America: Land use/ cover changes since 1987 in the Llanos Orientales of Colombia. Applied Geography. 32: 766-776.

Roy, D.B., Rothery, P., Moss, D., Pollard, E., Thomas, J.A. (2001). Butterfly numbers and weather: Predicting historical trends in abundance and the future effects of climate change. Journal of Animal Ecology. 70: 201-217.

Salinas, Y. (2011). Dinámicas en el mercado de la tierra en Colombia. Documento elaborado para la oficina Regional de la FAO para América Latina y el Caribe Home.

Sánchez-Cuervo, A.M., Aide, T.M. (2013). Identifying hotspots of deforestation and reforestation in Colombia (20012010): implications for protected areas. Ecosfera. 4: 21.

Sokal, R.R. \& Rohlf, F.J. (1979). Introducción a la bioestadística. Madrid. España. Reverte. p. 380.

Scott, J.A., Epstein, M.E. (1987). Factors affecting phenology in a temperate insect community. The American Midland Naturalist. 117: 103-118.

Shahabuddin, G., Terborgh, J.W. (1999). Frugivorous Butterflies in Venezuelan Forest Fragments: Abundance, Diversity and the Effects of Isolation. Journal of Tropical Ecology. 15: 703-722.
Shapiro, A.M. (1974). The butterfly fauna of the Sacramento Valley, California. Journal of the Lepidopterists Society. 13: $73-82$.

Shelly, T.E., Ludwig, D. (1985). Thermoregulatory behavior of the butterfly Calisto nubila (Satyridae) in a Puerto Rican forest. Oikos. 44: 229-233.

Sorto, R.E. (2013). Diversidad de mariposas diurnas en la reserve de la biósfera, Sierra Apaneca -Lamatepec, el Salvador. Revista Bioma. 1: 31-35.

Sparks, T.H., Yates, T.J. (1997). The effect of spring temperature on the appearance dates of British butterflies 1883-1993. Ecography. 20: 368-374.

Urbano, P., Munevar, J., Mahecha, J., Hincapie, E. (2014). Diversidad y estructura de las comunidades de lepidóptera en la zona del ecotono entre el piedemonte llanero y la sabana inundable en Casanare-Colombia (Lepidóptera: Papilionoidea). SHILAP Revista de Lepidopterología. 42: 433-437.

Valtonen, A., Molleman, F., Chapman, C.A., Carey., J.R., Ayres, M.P., Roininen, H. (2013). Tropical phenology: Biannual rhythms and interannual variation in an Afrotropical butterfly assemblage. Ecosphere. 4: 36

Vargas, M., Martínez, N., Gutiérrez, L., Prince, S., Herrera, V., Torres, L. (2011). Riqueza y abundancia de Hesperoidea y Papilionoidea (Lepidóptera) en la Reserva Natural Las Delicias, Santa Marta, Magdalena, Colombia. Acta Biológica Colombiana. 16: 43-60.

Villarreal, H., Álvarez, M., Córdoba, S., Escobar, F., Fagua, G., Gast, F., Mendoza, H., Ospina, M., Umaña, A. (2006). Manual de métodos para el desarrollo de inventarios de biodiversidad. Programa de Inventarios de Biodiversidad. Instituto de Investigación de Recursos Biológicos Alexander von Humboldt. Bogotá, Colombia: Segunda Edición. p. 236.

Warren, A. D., Davis, K. J., Stangeland, E. M., Pelham, J. P. \& Grishin, N. V. (2013). Illustrated Lists of American Butterflies. Fecha de consulta: enero-junio de 2018. Disponible en: http://www.butterfliesofamerica.com/

Wolda, H. (1988). Insect seasonality: Why? Ann. Rev. Ecol. Syst. 19: $1-18$. 